\title{
Inherent and apparent traits in microbial nutrient uptake
}

\author{
Dag L. Aksnes ${ }^{1, *}$, Francisco J. Cao ${ }^{2}$ \\ ${ }^{1}$ Department of Biology, University of Bergen, 5020 Bergen, Norway \\ ${ }^{2}$ Departamento de Física Atómica, Molecular y Nuclear, Universidad Complutense de Madrid, Ciudad Universitaria, \\ 28040 Madrid, Spain
}

\begin{abstract}
Nearly 50 yr ago, the Michaelis-Menten (MM) model, originally derived for enzyme kinetics, was adapted to characterize microbial nutrient uptake and has become a framework for defining microbial traits in competition theory, evolutionary dynamics, and ocean ecosystem models. We provide theoretical evidence that microbial traits and environmental properties are not appropriately distinguished in current ecological modeling that makes use of MM models, and we propose a framework where inherent microbial traits are explicitly distinguished from environmental variables. This provides novel expectations on how nutrient uptake is affected by cell size, porter density, temperature, and nutrient regimes, and we show that uptake kinetics and tradeoffs likely differ between oligotrophic and eutrophic regimes. We present mechanistic expressions for the affinity and the half-saturation $(K)$ coefficients, and our results suggest that $K$ might behave opposite to that commonly assumed in ecological modeling and should be abandoned as an index of uptake efficiency. Our results further suggest that the effect of organism size, considered a master trait in modeling, on specific uptake and growth rates is effectively modified by porter density, although differently in oligotrophic and eutrophic regimes. While nutrient uptake studies have commonly been carried out at a bulk scale, which is much larger than that experienced by the organisms, our study emphasize the need for observations at the scale of the individual.
\end{abstract}

KEY WORDS: Nutrient uptake $\cdot$ Microorganism $\cdot$ Trait $\cdot$ Affinity $\cdot$ Half-saturation constant $\cdot$ Model

\section{INTRODUCTION}

Microorganisms dominate the abundance, diversity and metabolic activity of the ocean and regulate important biogeochemical pathways that involve the global carbon cycle (Azam \& Malfatti 2007). Consequently, microbial processes are represented in numerical models used to analyze global carbon cycle-climate feedbacks and how marine ecosystems respond to environmental gradients in e.g. temperature, stratification, and nutrient regimes (Fasham et al. 1990, Boyd \& Doney 2002, Schmittner et al. 2005, Behrenfeld et al. 2006, Sarmiento \& Gruber 2006). Concerns have been raised against the aggregation of the microbial diversity into a few functional groups in such models (Anderson 2005, Follows et al. 2007, Franks 2009) and a 'trait-based' approach has recently emerged to increase the microbial resolution in ocean models (Follows et al. 2007, Litchman et al. 2007, 2010, Barton et al. 2010). A general challenge in ecological modeling, particularly in the trait-based approach, is how to parameterize organism traits, their plasticity, trade-off conflicts, and their relationships with environmental variables, such as temperature and nutrient regime. E.g. phytoplankton appear to have the ability to adjust their transporter systems according to the nutrient regime (Dyhrman \& Palenik 2001, Song \& 
Ward 2007, Franks 2009) and trait-based modeling acquires parameterization of such plasticity.

Ocean ecosystem modeling follows a general framework (e.g. Fasham et al. 1990, Sarmiento \& Gruber 2006) where the rate of change in tracers such as nutrients and phytoplankton are described by equations that account for transport (advection and mixing), sinking due to gravity, and biological sinks and sources. Omitting the transport terms, the local rate of change of a microbial state variable is expressed as $\mathrm{d} B / \mathrm{d} t=(g-m) B$, where $B$ is biomass, $t$ is time, $g$ is the source (instantaneous growth rate) and $m$ is the sink (instantaneous mortality rate). Here, $g=g_{\max } \times$ LIM where $g_{\max }$ is the maximum growth rate (commonly assumed to be temperature dependent) and LIM is a dimensionless quantity between 0 and 1 that expresses the degree of limitation from one or several environmental variables such as light and nutrients. If we consider just one limiting nutrient, represented by the ambient nutrient concentration $\left(S, \mathrm{~mol} \mathrm{~m}^{-3}\right)$, the standard representation of limitation (Monod 1949) is LIM $=S /\left(K_{\mathrm{g}}+\right.$ $S)$, where $K_{\mathrm{g}}\left(\mathrm{mol} \mathrm{m}^{-3}\right)$ is the half-saturation constant for growth. At the time scale of microbial doubling time, $\ln (2) / g$, growth and nutrient uptake rates are connected, and in ocean ecosystem models the halfsaturation constant for growth rate is commonly not differentiated (Sarmiento \& Gruber 2006, Franks 2009, Barton et al. 2010) from the half-saturation constant $(K)$ for nutrient uptake that is expressed in the Michaelis Menten (MM) equation (Michaelis \& Menten 1913, Briggs \& Haldane 1925, Dugdale 1967) for nutrient uptake rate $\left(V, \mathrm{~mol} \mathrm{~m}^{-3} \mathrm{~s}^{-1}\right)$ :

$$
V=V_{\max } \frac{S}{K+S}
$$

where $V_{\max }$ is the maximum nutrient uptake rate.

This equation has been applied extensively in experimental (Dugdale 1967), theoretical (Tilman 1981, Weitz et al. 2005, Armstrong 2008) and modeling (Sarmiento \& Gruber 2006, Franks 2009, Barton et al. 2010) studies over the last $50 \mathrm{yr}$ to describe the relationship between nutrient uptake rate and the ambient nutrient concentration. In ecological modeling, $K$ (the ambient nutrient concentration at half the maximum uptake rate) often appears as an invariant trait of a microbial species or of a functional group (Tilman 1981, Sarmiento \& Gruber 2006, Franks 2009, Barton et al. 2010). This is in contrast to $V_{\max }$ and $g_{\max }$ which are often assumed to be temperature dependent.

The ambient nutrient concentration is characterized by a single quantity $(S)$ in the MM model (Eq. 1), and $K$ is commonly measured in bulk experiments, i.e. where $S$ is measured at a scale much larger than that experienced by the organisms. Microorganisms, however, would in general be surrounded by a boundary layer with decreasing nutrient concentration towards the cell due to diffusion-limited flux through this layer (Munk \& Riley 1952, Jumars et al. 1993, Falkowski \& Oliver 2007). The net flux $(J)$ of nutrient molecules towards a spherical cell that is diffusion limited is expressed by (Pasciak \& Gavis 1974, Berg \& Purcell 1977, Jumars et al. 1993):

$$
J=4 \pi \operatorname{Dr}\left(S-S_{\mathrm{r}}\right)
$$

where $D\left(\mathrm{~m}^{2} \mathrm{~s}^{-1}\right)$ is the solute's molecular diffusion coefficient, $r$ is cell size (radius, $\mathrm{m}$ ), $S_{\mathrm{r}}$ is the nutrient concentration at the cell surface. Standard use of the MM equation in modeling, but also in experimental work, does not resolve the boundary layer, which means that $K$ is defined by $S$ instead of $S_{\mathrm{r}}$ as the reference concentration. An implication is that a measured $K$ will, in addition to microbial traits, reflect properties that determine the nutrient transport towards the cell, e.g. molecular diffusion and thereby temperature (Aksnes \& Egge 1991, Jumars et al. 1993). The assumption of invariant half-saturation constants for functional groups and species in models where environmental variables such as temperature are temporally and spatially heterogeneous might therefore be inappropriate (Aksnes et al. 1995, Smith 2010). Another related concern is that values of $K$ tend to vary substantially, both in experiments (Harrison et al. 1989, Button et al. 2004) and in models (Franks 2009). In 10 recently published ecosystem models, Franks (2009) found that variation of $>2$ orders of magnitude in $K$ was applied for similar functional groups of phytoplankton, and questioned whether the MM equation is the best representation of nutrient uptake in ecosystem models.

There have been several studies (Pasciak \& Gavis 1974, Armstrong 2008, see also references in Jumars et al. 1993) where the MM model has been combined with diffusion limitation to describe microbial nutrient acquisition. The model of Pasciak \& Gavis (1974) (PG), which was revisited by Armstrong (2008), assumes the modified MM equation for the nutrient uptake rate: $V=V_{\max } \times S_{\mathrm{r}} /\left(K_{\mathrm{r}}+S_{\mathrm{r}}\right)$ where $K_{\mathrm{r}}$ is the half-saturation constant with $S_{\mathrm{r}}$ rather than $S$ as the reference concentration. Since the flux $J$ (Eq. 2) corresponds to $V$, equating $V$ with $J$ provides the quadratic expression that connects $S_{\mathrm{r}}$ and $S$ : $S_{\mathrm{r}}^{2}+\left(V_{\max } /(4 \pi D r)+K_{\mathrm{r}}-S\right) S_{\mathrm{r}}-K_{\mathrm{r}} S=0$. This result has not been utilized in ecosystem modeling, and Armstrong (2008) suggested that this might be due 
to the inconvenient algebraic expression for $V$. Another concern is that the mechanistic interpretation of $K_{\mathrm{r}}$, like $K$, is not trivial. $K_{\mathrm{r}}$ is according to Armstrong (2008) given by $K_{\mathrm{r}}=(a h)^{-1}\left(K_{\mathrm{r}}\right.$ is denoted $k_{\mathrm{p}}$ in Eq. 2 of Armstrong 2008) where $a$ is the acquisition rate of a porter at the cell surface and $h$ the time period that is needed for a porter to absorb a nutrient molecule. The acquisition rate can be further decomposed (Aksnes \& Egge 1991, Armstrong $2008)$ into the area of a porter $\left(A, \mathrm{~m}^{2}\right)$ and a velocity component $\left(v, \mathrm{~m} \mathrm{~s}^{-1}\right)$. This velocity component corresponds to a mass transfer coefficient (Munk \& Riley 1952, Aksnes \& Egge 1991) that is affected by both microbial traits and environmental properties in a way that is not straightforward.

Here, we combine results from previous studies (Berg \& Purcell 1977, Zwanzig 1990, Aksnes \& Egge 1991) to derive a non-MM nutrient uptake model. The inherent traits of our model correspond to those introduced by Aksnes \& Egge (1991). The substrate concentration assumed in that study, however, corresponds to the concentration at the cell surface (i.e. $S_{\mathrm{r}}$ ) rather than the bulk concentration $(S)$ that is commonly observed in uptake experiments and assumed in ecological modeling. We propose a framework where nutrient uptake rate is derived as a function of $S$ and where inherent microbial traits are explicitly distinguished from environmental properties. In this framework both affinity and the half-saturation coefficients emerge as apparent microbial traits. Finally, we present and discuss novel predictions on how nutrient uptake is affected by organism size, porter density, temperature, and nutrient regime.

\section{THE MODEL}

We consider microbial uptake for 2 idealized nutrient regimes: the oligotrophic situation where the ambient nutrient concentration is low $(S \rightarrow 0)$ and the eutrophic situation that is high in nutrients $(S \rightarrow \infty)$. We present a mechanistic model where the uptake rate is a function of $S$ as in the MM equation, but with a different functional form. Throughout this study, we assume a spherical microorganism that lives in a medium where the transport of nutrient molecules is governed by molecular diffusion (Berg \& Purcell 1977, Jumars et al. 1993, Falkowski \& Oliver 2007) as expressed in Eq. (2). The analysis can be extended to non-spherical forms (Munk \& Riley 1952, Armstrong 2008) but this is not considered in the present study.

\section{Uptake rate in the oligotrophic regime $(S \rightarrow 0)$}

The flux $(J)$ of Eq. (2) equals the uptake rate, and the maximum diffusion-limited uptake rate, $V=$ $J_{\text {max }}=4 \pi D r S$, is obtained for $S_{\mathrm{r}}=0$ (Jumars et al. 1993). This corresponds to a perfect sink where all encountered nutrient molecules are absorbed immediately. For such behavior the uptake rate can be modeled $V=\alpha_{\max } S$, where $\alpha_{\max }=4 \pi D r\left(\mathrm{~m}^{3} \mathrm{~s}^{-1}\right)$ is a nutrient 'clearance rate' that is termed the maximum affinity (Thingstad et al. 2005, Tambi et al. 2009). Microbes, however, are not likely to be perfect sinks. In the case of active uptake through porters at the cell surface, only a fraction of the surface is covered by such porters (Berg \& Purcell 1977, Jumars et al. 1993, Button et al. 2004). In the case of diffusion limitation, theoretical studies have shown that the chance of capturing a nutrient molecule is a non-linear function of the porter number, and the flux can be expressed as (Berg \& Purcell 1977, Zwanzig 1990):

$$
J=J_{\max } \frac{n s}{n s+\pi r(1-p)}=4 \pi D r S \frac{n s}{n s+\pi r(1-p)}
$$

where $n$ is the number of porters of the cell, $s$ is the porter size (radius, $\mathrm{m})$, and the factor $(1-p)$, where

$$
p=\frac{n \pi s^{2}}{4 \pi r^{2}}
$$

was introduced (Zwanzig 1990) to correct for interaction between porters.

We obtain an expression for the uptake affinity $(\alpha$, $\mathrm{m}^{3} \mathrm{~s}^{-1}$ ) by dividing Eq. (3) by $S$. In contrast to $\alpha_{\max }$ this affinity resolves 2 microbial traits in addition to cell size, i.e. the porter number and size:

$$
\alpha=4 \pi D r \frac{n s}{n s+\pi r(1-p)}
$$

Eq. (3) assumes that porters are always receptive for uptake and therefore, like Eq. (2), predicts no saturation in uptake rate with increasing $S$. This is reasonable for low nutrient concentrations when the time period between nutrient encounters at a porter is much longer than the time it takes to absorb the nutrient molecule. Thus, for the oligotrophic regime $(S \rightarrow 0), V$ can be approximated by:

$$
\mathrm{V}_{(S \rightarrow 0)}=\alpha S
$$

More accurately this equation is valid for $S_{\mathrm{r}} \rightarrow 0$ which is less strict than $S \rightarrow 0$. 

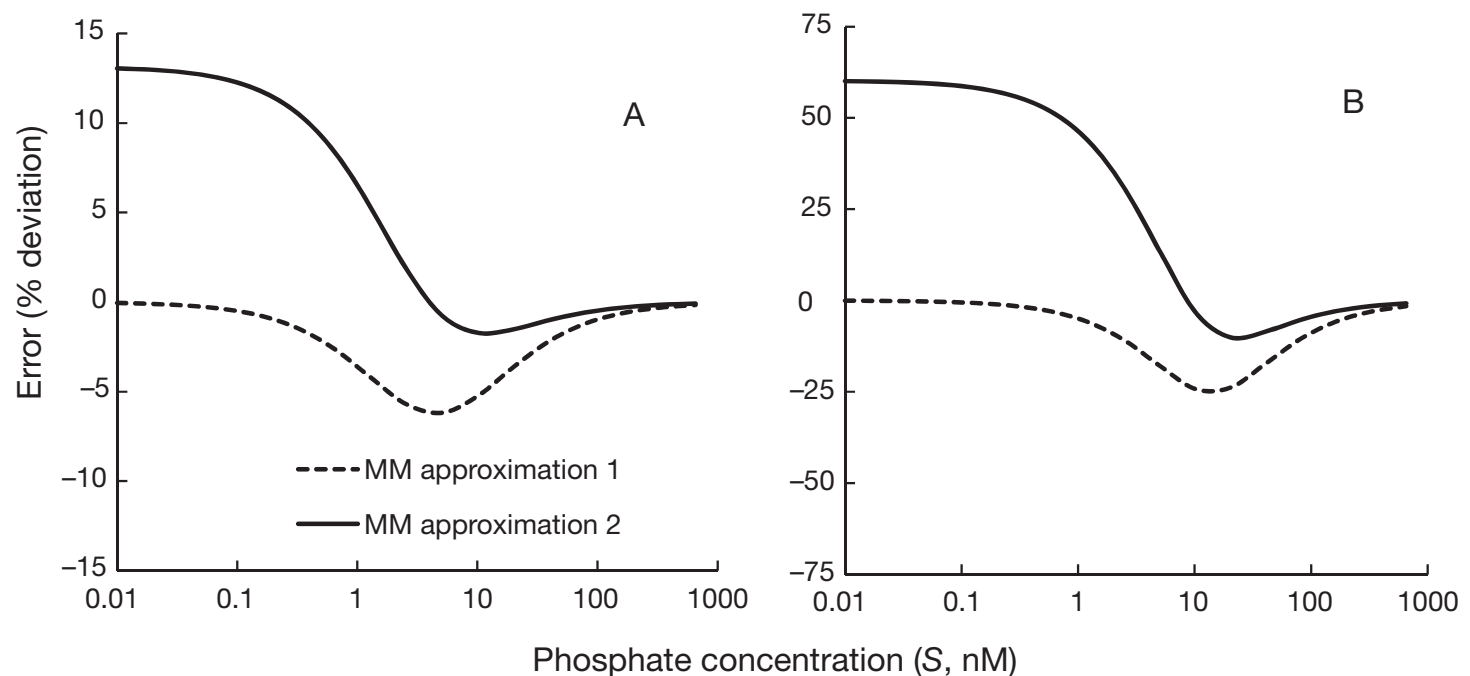

Fig. 1. Error of 2 Michaelis-Menten (MM) approximations at different phosphate concentrations (S). MM approximation 1 assumes $K=V_{\max } / \alpha$, while MM approximation 2 assumes the $K$ of the uptake model in Appendix 1. Calculations are based on the assumed characteristics (A) of Vibrio splendidus (see Table 1) and (B) for a porter density that has been increased 10 times. The error represents the deviation from the uptake rate calculated with the analytical expression for uptake rate given in Appendix 1

increased porter density, with errors that might exceed $50 \%$ when porter density is increased 10 times (Fig. 1B). At low porter densities $(n s \ll \pi r)$, the errors of the MM approximations diminish (not shown). As will be discussed below, however, this result does not warrant the assumption of invariant $K$, independency of $V_{\max }$, and the extrapolation of measured $K$ values to environmental conditions other than those occurring in the particular experiment.

Using the estimated traits of Vibrio splendidus (Table 1) as baseline, we now present results on how porter number, microbial size, and temperature are expected to affect nutrient uptake in the oligotrophic and the eutrophic regimes.

\section{Effects of porter number and organism size on uptake rate}

For an organism of a particular size, our results suggest that the benefit of increased porter number is very different for organisms living in oligotrophic (when $\alpha$ is rate limiting) and eutrophic (when $V_{\max }$ is rate limiting) environments (Fig. 2). This is because $\alpha$ saturates for large $n$ (Eq. 4), and $V_{\max }$ increases proportionally to $n$ (Eq. 6).

Fig. 3 shows how different combinations of porter density and organism size are expected to affect the specific uptake rate $(V / Q$, where $Q$ is the biomass of the cell as defined in Table 1), which is a proxy for growth rate. Each line represents a given porter den-

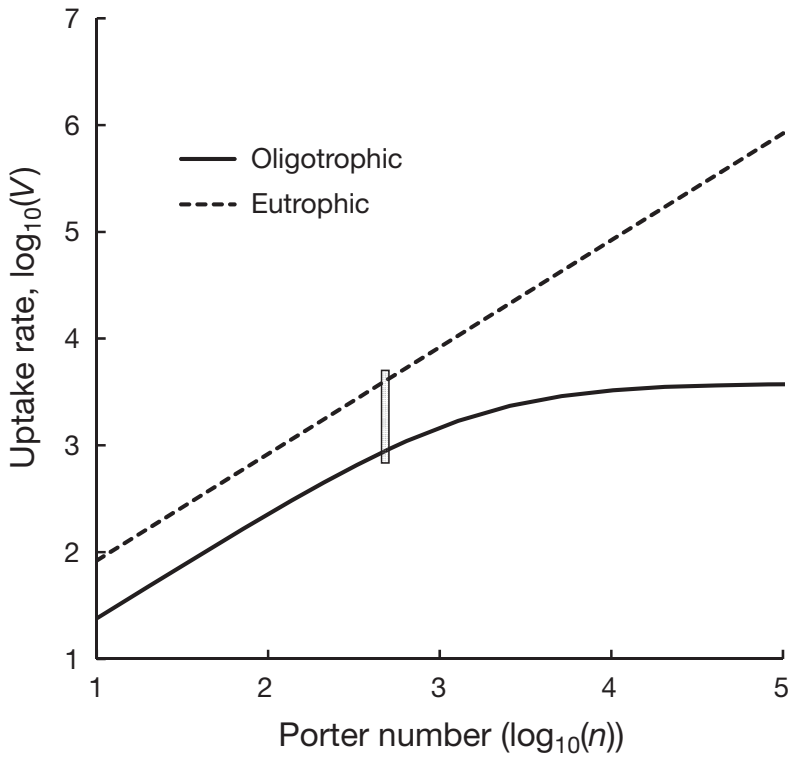

Fig. 2. Variation in uptake rate $\left(V\right.$, molecules $\left.\mathrm{s}^{-1}\right)$ shown as a function of porter number $(n)$ in the oligotrophic and the eutrophic condition (see 'The model'). The shaded area indicates the range of Vibrio splendidus (Table 1)

sity (i.e. $n / r^{2}$ is constant) and indicates a decrease in specific uptake rate with increased size $(r)$, but where the actual slope depends on the nutrient regime. It also shows that the size-related decrease in specific uptake rate is counteracted by increased porter density, and that this compensation is much more efficient in the eutrophic than in the oligotrophic situation. Such combined effects of porter density, size 

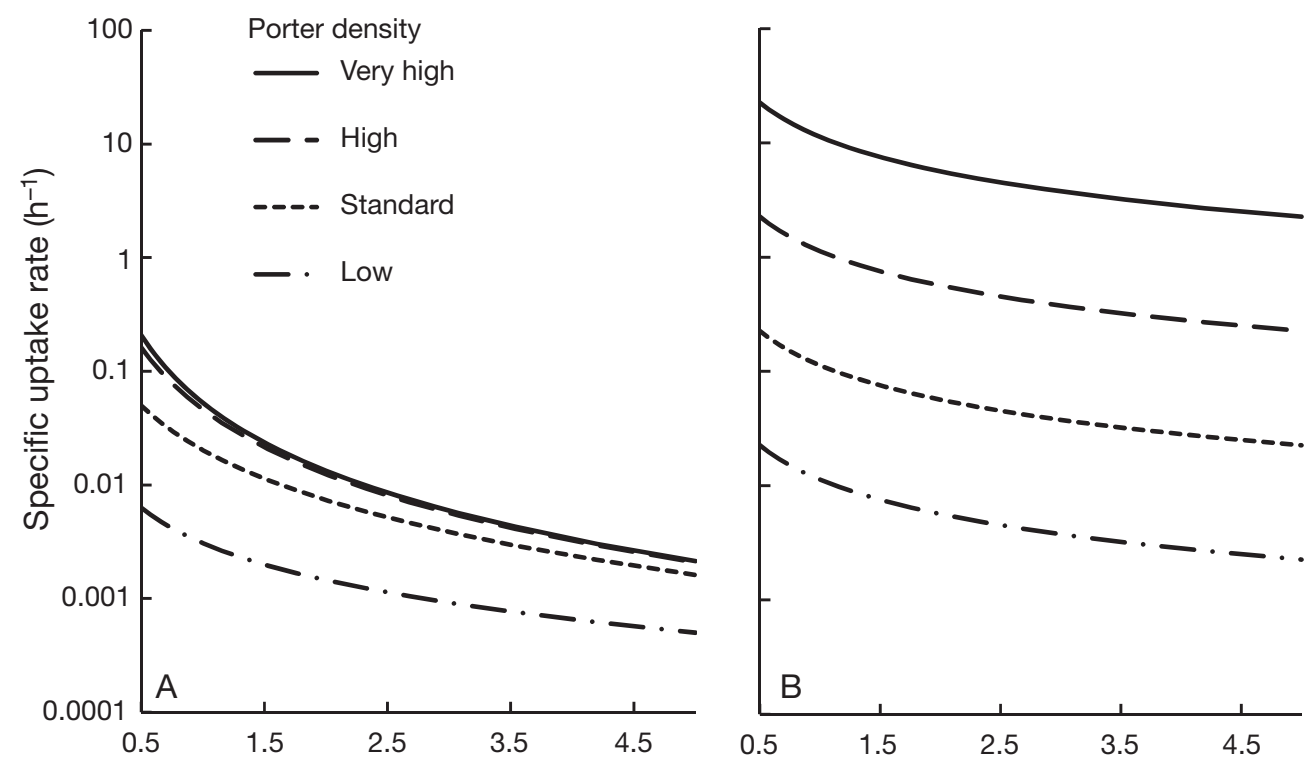

Fig. 3. Variation in specific uptake rate $(V / Q$, where $Q$ is cellular biomass) shown as a function of cell size $(r)$ at 4 different porter densities in the (A) oligotrophic and (B) eutrophic condition (see 'The model'). The standard porter density corresponds to $0.048 \%$ of the surface area which is the estimate for Vibrio splendidus (Table 1). Low, high and very high is $0.1,10$ and 100 times the standard porter density, respectively

Size $(r, \mu \mathrm{m})$

and nutrient regime on uptake rates and ultimately on the competitive ability of species are not readily resolved within the MM framework.

\section{Dual effect of temperature on uptake rate}

Temperature is not explicitly expressed in our model, but temperature affects molecular diffusion and the cellular machinery. Molecular diffusion is affected by temperature with a $Q_{10}$ (the ratio between rates obtained at 2 temperatures that differ by $10^{\circ} \mathrm{C}$ ) of about 1.4 around $10^{\circ} \mathrm{C}$ for rapidly diffusing ions (Jumars et al. 1993). According to Eq. (4) this particular effect of temperature on molecular diffusion will also affect affinity. In addition, for temperatures lower than the optimal temperature, increased temperature increases the growth rate, and a $Q_{10}$ close to 2 has been found for phytoplankton maximum growth rate (Eppley 1972, Bissinger et al. 2008). We can express this temperature effect through $V_{\text {max }}$ or equivalently through the handling time, i.e. increasing the temperature causes an increase in $h^{-1}$ (Eq. 6) with a $Q_{10}$ of 2 . The dual effect of temperature leads to the expectation that the uptake rate for an organism should respond differently to temperature changes in oligotrophic and eutrophic environments (Fig. 4). Such interactions between the temperature and the nutrient regime are not contained in the MM model unless $K$ is phenomenologically assumed to vary with temperature (in a different way than $V_{\max }$ ).

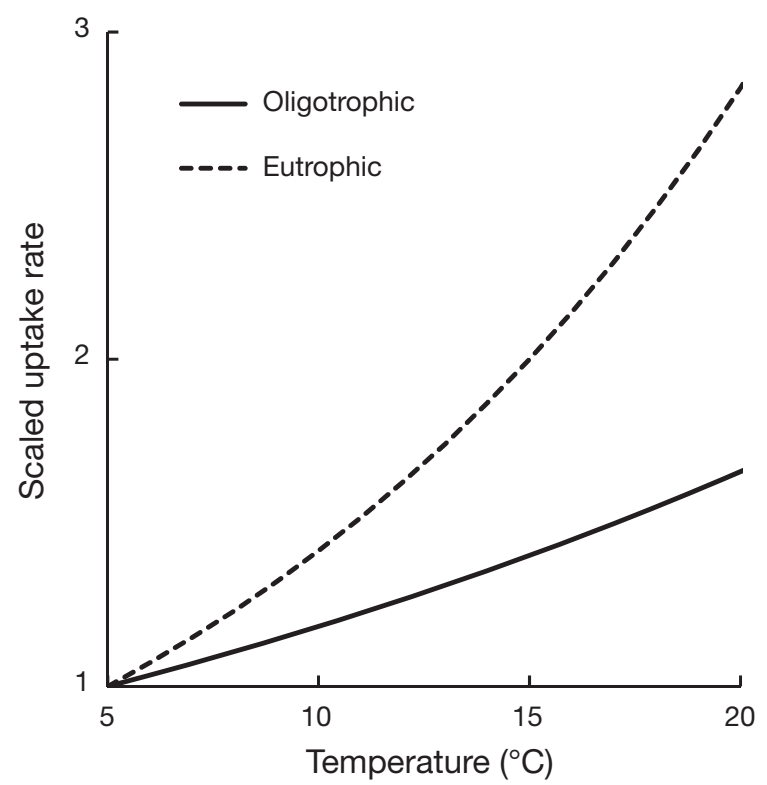

Fig. 4. Variation in scaled uptake rate $\left(V_{\text {at } T} / V_{\text {at } 5{ }^{\circ} \mathrm{C}}\right)$ shown as a function of temperature $(T)$ in the oligotrophic and the eutrophic condition (see 'The model'). The parameter values were chosen as for $V$. splendidus (Table 1), but where molecular diffusion $(D)$ and the reciprocal porter handling time $\left(h^{-1}\right)$ were assumed to be affected by temperature according to $Q_{10}$-values of 1.4 and 2.0 , respectively

\section{Half-saturation constant and uptake efficiency increase with porter number}

The analytical expression for the non-MM half-saturation constant (see Appendix 1) provides testable predictions on how $K$ is expected to vary with prop- 


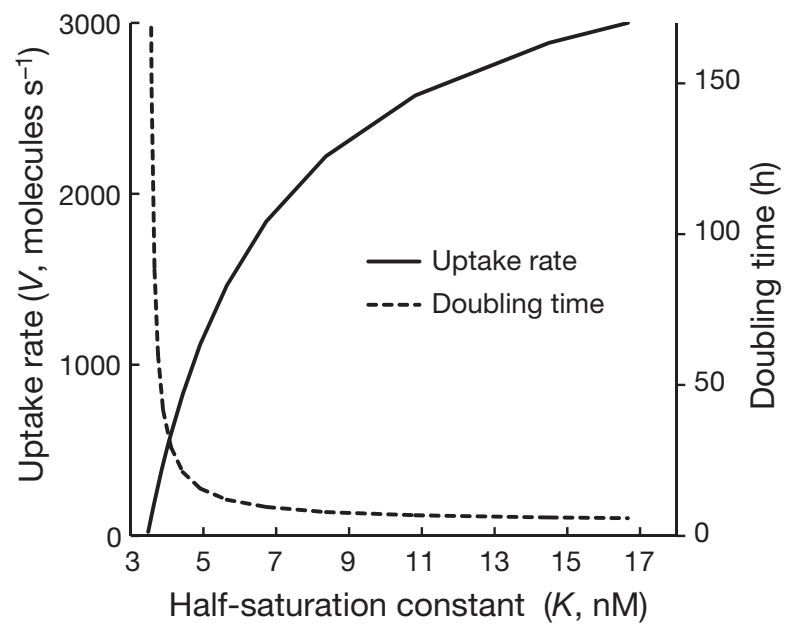

Fig. 5. Relationship between uptake rate $(V)$ and halfsaturation constant $(K)$, showing an apparent paradox: In contrast to what is commonly assumed, $V$ increases with increased $K$ in the oligotrophic condition (see 'The model'). The indicated span in $K$ (here defined as $K=V_{\max } / \alpha$ ) corresponds to porter numbers $(n)$ ranging from 10 to 6000 . The indicated doubling time is the time it takes to absorb the phosphate quantity that corresponds to the cellular phosphate content $(Q)$ of Vibrio splendidus (Table 1)

erties of the organism and the environment. Contrary to the common interpretation, $K$ can-according to this expression - increase in parallel with increased uptake efficiency at a low nutrient concentration. Increased porter number $(n)$ means increased uptake efficiency, and because $K$ increases with increased porter number, $K$ increases in parallel with increased uptake efficiency. The same also applies to the MM half-saturation (which is given by $K=V_{\max } / \alpha$ ) (Fig. 5).

\section{DISCUSSION}

In a recent review, Azam \& Malfatti (2007, p. 789) stated: 'Microbial oceanography is a field that is caught between scales - microbial processes must be understood at the scale of the individual microorganism, but yet we want to understand the cumulative influence of microbial processes on the ocean as a biogeochemical system'. This multi-scale challenge is particularly true for ocean ecosystem modeling. Microorganisms interact with the ocean system at the nanometer to the millimeter scale and processes at these scales can obviously not be explicitly resolved in models that couple microbial processes and global ocean circulation models. Parameterizations are nevertheless required, and it is important that they are consistent with the small-scale microbial processes.
While the pioneering studies of Michaelis \& Menten (1913) and Briggs \& Haldane (1925) represent a milestone in the history of enzyme kinetics as the foundation of the steady-state treatment of enzymecatalyzed reactions (Baici 2006), we argue that progress in microbial ecological modeling will benefit from inclusion of microbial nutrient acquisition traits beyond those offered by the current MM framework. Such increased trait resolution also provides a mechanistic interpretation of the MM halfsaturation constant, suggesting some constraints on its use in a modeling context.

\section{The half-saturation constant is redundant as an index of uptake efficiency}

Our results suggest that $K$ likely increases with porter density, which means increased rather than decreased uptake efficiency (Fig. 5). This suggests that the common interpretation of $K$ as an index for uptake efficiency at low nutrient concentrations is misleading. But it is also redundant because $\alpha$ by definition (Eq. 4) is more appropriate (Healey 1980, Aksnes \& Egge 1991, Button et al. 2004), which suggests that affinity-based uptake models (Aksnes et al. 1995, Smith et al. 2009, Thingstad et al. 2010) might be preferable in ecological modeling. It can be noted that affinity $(\alpha)$, according to Eq. (4), facilitates inclusion of a simpler temperature dependency than $K$. This is because $\alpha$ reflects temperature through molecular diffusion, while $K$ reflects temperature through both handling time and molecular diffusion.

\section{Apparent and inherent traits}

Both $K$ and $\alpha$ are affected by processes taking place outside the organism through the solute's molecular diffusion coefficient, and these quantities are therefore expected to change without any change in organism traits, which clearly means that they are not inherent to the organism. In oceanography, the differentiation between apparent and inherent optical properties is of fundamental importance. We recommend a similar differentiation to be made between apparent and inherent microbial traits. Our results suggest that while $K$ and $\alpha$ classify as apparent traits, the quantities $r, n, s$, and possibly $h$ are inherent traits. Organism traits are characterized by plasticity, and a priority in biology is to characterize and understand such plasticity. To achieve this, inherent traits are needed. Furthermore, these are 
required in the analyses of trait trade-off conflicts and to provide a mechanistic base for e.g. prediction of nutrient uptake rate. In contrast, apparent traits are obviously useful to characterize the outcome of experiments and to derive estimates of inherent traits, as illustrated in Table 1. Similar to the apparent optical properties (such as the attenuation for downwelling irradiance), apparent traits might under certain circumstances also be useful as parameters in ecological models. The current modeling practice of assigning invariant half-saturation constants for variable environmental conditions (i.e. by extrapolation), however, needs to be revised.

\section{Organism size as master trait and the modifying effect of porter density}

Numerous functional traits and core metabolic rates correlate with size, and organism size has therefore been termed a master trait (Litchman et al. 2010). Our results demonstrate that the role of size is modified by porter density (i.e. the fraction of surface area covered with porters).

First, we look at the assumption of constant porter density across cell size (i.e. $n / r^{2}$ is constant). If cellular volume is taken as a proxy for $Q$, a rapid quadratic drop in the mass specific uptake rate with increased size $\left(V / Q \propto r^{-2}\right)$ is expected in the diffusion limited (i.e. oligotrophic) case (Eqs. 2 \& 5). However, cells limited by a low porter number, as might be the case in eutrophic conditions (Eq. 6), are expected to have a slower drop in the mass-specific uptake rate as size increases $\left(V / Q \propto r^{-1}\right)$. This suggests that the actual effect of cell size on specific uptake rate depends on the nutrient regime, and that the 'penalty' of increased size is lower in eutrophic than in oligotrophic regimes.

Because increased porter density might reduce the penalty of cell size becoming large, our analysis questions the adaptive value of constant porter density across cell size. Generally, the benefit of increased porter density is expected to decrease and ultimately cease when porter capacity exceeds the processing capacity of the growth machinery. Larger cells, however, are expected to benefit more from a high porter density than smaller cells, because one unit of biomass of a large cell has less surface area available to support its biomass with nutrients. A decrease in $h$, which appears likely for cellular machineries that are in demand of nutrients, results in a similar effect as the increase in porter number.

Jumars et al. (1993) suggested that the foraging consequence of the high efficacy of a low porter density is that the marginal gain per porter rapidly drops off as the plateau predicted by Eq. (3) is approached for large $n$, and that the optimal porter density is therefore likely to be low. Our analysis suggests that although this might be true for the oligotrophic case it is not generally true for all $S$. For the eutrophic case, Eq. (6) suggests that there is no drop at all in the marginal gain per porter.

Estimation of the optimal porter number requires not only the gain but also the cost of porters such as energy expenditure and competition for space. Their potential role as entrance point for viral attacks (Weitz et al. 2005, Yooseph et al. 2010) is of particular interest as increased mortality risk might tend to reduce the optimal porter number below the level expected without such risk. Furthermore, trade-offs involving porter density are likely to be affected by the nutrient regime and cell size as indicated above (Fig. 3).

\section{Need for observations at the scale of the cell}

Mechanistic theory is useful, but observations and experiments are obviously needed to characterize cellular traits and understand their plasticity and trade-offs, and how these translate into nutrient uptake and growth. Certain aspects of the theory presented here can be tested in bulk experiments, such as the expectation that the affinity and the halfsaturation constants (Table 1) respond differently to temperature. Microbial processes must be understood at the scale of the individual microorganism (Azam \& Malfatti 2007), however, and we hope our analysis will stimulate studies that aim at resolving processes and traits at this scale. From such knowledge we should, at least in principle, be able to make predictions of the uptake rates and the parameters that we observe at the bulk scale. Collaborations of cell biologists and ecologists to design experiments with concurrent measurements of inherent traits and nutrient uptake rates would likely be an important step forward.

Acknowledgements. We thank T. F. Thingstad for recurrent and stimulating discussions on microbial nutrient uptake during the last 2 decades. Thanks also to A. D. Barton, S. Eliassen, Ø. Fiksen, J. Giske, and R. Rosland for valuable comments on the manuscript. F.J.C. acknowledges funding from NILS mobility project (EEA-EFTA-UCM, NorwaySpain) through Abel Extraordinary Chair, from MEC (Spain) through project FIS2010-17440, and from UCM and CM (Spain) through project GR35/10-A-920GR35-10-A-911. 


\section{LITERATURE CITED}

Aksnes DL, Egge JK (1991) A theoretical model for nutrient uptake in phytoplankton. Mar Ecol Prog Ser 70:65-72

Aksnes DL, Ulvestad KB, Balino BM, Berntsen J, Egge JK, Svendsen E (1995) Ecological modeling in coastal waters: towards predictive physical-chemical-biological simulation-models. Ophelia 41:5-36

Anderson TR (2005) Plankton functional type modelling: running before we can walk? J Plankton Res 27:10731081

Armstrong RA (2008) Nutrient uptake rate as a function of cell size and surface transporter density: a Michaelis-like approximation to the model of Pasciak and Gavis. DeepSea Res I 55:1311-1317

Azam F, Malfatti F (2007) Microbial structuring of marine ecosystems. Nat Rev Microbiol 5:782-791

- Baici A (2006) Enzyme kinetics: the velocity of reactions. Biochem J (BJ Classics), c15-c15 doi:10.1042/BJ2006c015

> Barton AD, Dutkiewicz S, Flierl G, Bragg J, Follows MJ (2010) Patterns of diversity in marine phytoplankton. Science 327:1509-1511

- Behrenfeld MJ, O'Malley RT, Siegel DA, McClain CR and others (2006) Climate-driven trends in contemporary ocean productivity. Nature 444:752-755

Berg HC, Purcell EM (1977) Physics of chemoreception. Biophys J 20:193-219

> Bissinger JE, Montagnes DJS, Sharples J, Atkinson D (2008) Predicting marine phytoplankton maximum growth rates from temperature: improving on the Eppley curve using quantile regression. Limnol Oceanogr 53:487-493

> Boyd PW, Doney SC (2002) Modelling regional responses by marine pelagic ecosystems to global climate change. Geophys Res Lett 29, 1806, doi:10.1029/2001GL014130

> Briggs GE, Haldane JBS (1925) A note on the kinetics of enzyme action. Biochem J 19:338-339

Button DK, Robertson B, Gustafson E, Zhao XM (2004) Experimental and theoretical bases of specific affinity, a cytoarchitecture-based formulation of nutrient collection proposed to supercede the Michaels-Menten paradigm of microbial kinetics. Appl Environ Microbiol 70: 5511-5521

> Dugdale RC (1967) Nutrient limitation in the sea: dynamics, identification and significance. Limnol Oceanogr 12: 685-695

> Dyhrman ST, Palenik B (2001) A single-cell immunoassay for phosphate stress in the dinoflagellate Prorocentrum minimum (Dinophyceae). J Phycol 37:400-410

Eppley RW (1972) Temperature and phytoplankton growth in the sea. Fish Bull 70:1063-1085

- Falkowski PG, Oliver MJ (2007) Mix and match: how climate selects phytoplankton. Nat Rev Microbiol 5: 813-819

Fasham MJR, Ducklow HW, McKelvie SM (1990) A nitrogen-based model of plankton dynamics in the oceanic mixed layer. J Mar Res 48:591-639

Follows MJ, Dutkiewicz S, Grant S, Chisholm SW (2007) Emergent biogeography of microbial communities in a model ocean. Science 315:1843-1846

- Franks PJS (2009) Planktonic ecosystem models: perplexing parameterizations and a failure to fail. J Plankton Res 31: 1299-1306

> Harrison PJ, Parslow JS, Conway HL (1989) Determination of nutrient uptake kinetic parameters: a comparison of methods. Mar Ecol Prog Ser 52:301-312
Healey FP (1980) Slope of the Monod equation as an indicator of advantage in nutrient competition. Microb Ecol 5: 281-286

Jumars PA, Deming JW, Hill PS, Karb-Boss L, Yager PL, Dade WB (1993) Physical constraints on marine osmotrophy in an optimal foraging context. Mar Microb Food Webs 7:121-159

- Litchman E, Klausmeier CA, Schofield OM, Falkowski PG (2007) The role of functional traits and trade-offs in structuring phytoplankton communities: scaling from cellular to ecosystem level. Ecol Lett 10:1170-1181

- Litchman E, Pinto PD, Klausmeier CA, Thomas MK, Yoshiyama K (2010) Linking traits to species diversity and community structure in phytoplankton. Hydrobiologia 653:15-28

Michaelis L, Menten ML (1913) Die Kinetik der Invertinwirkung. Biochem Z 49:333-369

> Monod J (1949) The growth of bacterial cultures. Annu Rev Microbiol 3:371-394

Munk WH, Riley GA (1952) Absorption of nutrients by aquatic plants. J Mar Res 11:215-240

> Pasciak WJ, Gavis J (1974) Transport limitation of nutrient uptake in phytoplankton. Limnol Oceanogr 19:881-898

Sarmiento JL, Gruber N (2006) Ocean biogeochemical dynamics. Princeton University Press, Princeton, NJ

Schmittner A, Oschlies A, Giraud X, Eby M, Simmons HL (2005) A global model of the marine ecosystem for longterm simulations: sensitivity to ocean mixing, buoyancy forcing, particle sinking, and dissolved organic matter cycling. Global Biogeochem Cycles 19, GB3004, doi:10. 1029/2004GB002283

Smith SL (2010) Untangling the uncertainties about combined effects of temperature and concentration on nutrient uptake rates in the ocean. Geophys Res Lett 37 , L11603, doi:10.1029/2010GL043617

> Smith SL, Yamanaka Y, Pahlow M, Oschlies A (2009) Optimal uptake kinetics: physiological acclimation explains the pattern of nitrate uptake by phytoplankton in the ocean. Mar Ecol Prog Ser 384:1-12

Song B, Ward BB (2007) Molecular cloning and characterization of high-affinity nitrate transporters in marine phytoplankton. J Phycol 43:542-552

Tambi H, Flaten GAF, Egge JK, Bodtker G, Jacobsen A, Thingstad TF (2009) Relationship between phosphate affinities and cell size and shape in various bacteria and phytoplankton. Aquat Microb Ecol 57:311-320

Thingstad TF, Krom MD, Mantoura RFC, Flaten GAF and others (2005) Nature of phosphorus limitation in the ultraoligotrophic eastern Mediterranean. Science 309: 1068-1071

Thingstad TF, Strand E, Larsen A (2010) Stepwise building of plankton functional type (PFT) models: a feasible route to complex models? Prog Oceanogr 84:6-15

Tilman D (1981) Tests of resource competition theory using four species of Lake Michigan algae. Ecology 62:802-815

- Weitz JS, Hartman H, Levin SA (2005) Coevolutionary arms races between bacteria and bacteriophage. Proc Natl Acad Sci USA 102:9535-9540

Yooseph S, Nealson KH, Rusch DB, McCrow JP and others (2010) Genomic and functional adaptation in surface ocean planktonic prokaryotes. Nature 468:60-66

Zwanzig R (1990) Diffusion-controlled ligand-binding to spheres partially covered by receptors: an effective medium treatment. Proc Natl Acad Sci USA 87: 5856-5857 
Appendix 1. The first part, including Eqs. (A1) to (A4), summarizes the model for nutrient uptake and how affinity, maximal uptake rate, and the half-saturation constant relate to this model. The second part gives the derivation of the nutrient uptake model expressed in Eq. (A1)

\section{Model summary}

The analytical expression for nutrient uptake rate $(V$, mol s${ }^{-1}$ ) of a spherical cell where encounters with nutrient molecules are governed by molecular diffusion.

$$
V=\frac{b}{2 a}\left(1-\sqrt{1-\frac{4 a}{b^{2}}}\right)
$$

where

$$
a=\frac{h}{4 \pi D r S n}\left(1-\frac{\pi r p}{n s}\right), \quad b=\frac{1}{\alpha S}+\frac{h}{n}, \quad p=\frac{n \pi s^{2}}{4 \pi r^{2}}
$$

Affinity and approximation for the uptake rate in the oligotrophic case. For low nutrient concentrations (i.e. $S \rightarrow 0$ ) this expression reduces to $V_{S \rightarrow 0}=\alpha S$ where $\alpha$ is the affinity:

$$
\alpha=4 D \pi r \frac{n s}{n s+\pi r(1-p)}
$$

Maximal uptake rate and approximation for the uptake rate in the eutrophic case. For high nutrient concentrations (i.e. $S \rightarrow \infty$ ) this expression reduces to $V_{S \rightarrow \infty}=n / h$, i.e:

$$
V_{\max }=n / h
$$

The non-MM half-saturation constant. The nutrient concentration $(K)$ where uptake equals $50 \%$ of the maximal uptake rate (i.e. $n /(2 h)$ ) is:

$$
K=\frac{\pi r(2-p)+n s}{8 h \pi D r s}
$$

\section{Derivation of Eq. (A1)}

Berg \& Purcell (1977) derived an equation for the total flux $(J)$, which corresponds to the uptake rate $(V)$, of nutrient molecules towards the porters of a spherical cell. They considered the case where all porters are always receptive for uptake. In this case, the flux is equal to the encounter rate $(e)$ between nutrient molecules and all porters. In the case of non-zero handling time ( $h$ ) (Aksnes \& Egge 1991), however, the encounter rate is not equal to the flux, and below we will consider this situation. The Berg \& Purcell (1977) result gives the following equation for the encounter rate:

$$
e=e_{\max } \frac{n s}{n s+\pi r}
$$

where $e_{\max }=4 \pi D r S$. This equation was modified by Zwanzig (1990) to better fit numerical results for the case of high porter coverage. Zwanzig introduced a porter interaction term that is given by the fraction of the sphere's surface covered by porters, $p=\frac{n \pi s^{2}}{4 \pi r^{2}}$, and arrived at the modified Berg \& Purcell equation:

$$
e_{\mathrm{p}}=e_{\max } \frac{n s}{n s+\pi r(1-p)}
$$

However, for non-zero handling time the porters are closed during the handling time $h$, so that only nutrient molecule encounters with open porters are taken up by the cell and should be accounted for. The average number of open porters is $n_{0}=n-V h$, and the encounter rate for all the open porters is:

$$
e_{\mathrm{po}}=e_{\max } \frac{n_{\mathrm{o}} s}{n_{\mathrm{o}} s+\pi r\left(1-p_{\mathrm{o}}\right)}
$$

with

$$
p_{\mathrm{o}}=\frac{n_{\mathrm{o}} \pi s^{2}}{4 \pi r^{2}}=p\left(1-\frac{h}{n} V\right)
$$

As $\frac{e_{\mathrm{po}}}{n_{\mathrm{o}}}$ is the encounter rate for one open porter, the average time until the next encounter is $t_{\mathrm{e}}=\frac{n_{\mathrm{o}}}{e_{\mathrm{po}}}$, and afterward the porter will be closed during a time equal to the handling time $h$. Therefore, each porter will have an encounter after an average time of $t_{\mathrm{e}}+h$, and because there are $n$ porters the average nutrient uptake rate is given by $V=\frac{n}{t_{\mathrm{e}}+h}$.

However, as $t_{\mathrm{e}}$ depends on $V$ as

$t_{\mathrm{e}}=\frac{n_{\mathrm{o}}}{e_{\mathrm{po}}}=\frac{n}{e_{\mathrm{p}}}-\frac{V h}{e_{\max }}\left(1-\frac{\pi r p}{n s}\right)$,

this gives the following equation for the uptake rate of the cell:

$$
V=\frac{1}{\frac{1}{e_{\mathrm{p}}}+\frac{h}{n}\left[1-\frac{V}{e_{\max }}\left(1-\frac{\pi r p}{n s}\right)\right]}
$$

This implicit equation for $V$ can be rewritten as $a V^{2}-b V+1=0$ with $a=\frac{h}{n e_{\max }}\left(1-\frac{\pi r p}{n s}\right)$ and $b=\frac{1}{e_{\mathrm{p}}}+\frac{h}{n}$ which can be exactly solved for $V$ giving:

$$
V=\frac{b-\sqrt{b^{2}-4 a}}{2 a}=\frac{b}{2 a}\left(1-\sqrt{1-\frac{4 a}{b^{2}}}\right)
$$

which corresponds to Eq. (A1). The uptake rate can be given as a function of the nutrient concentration $(S)$, as $S$ is contained in $e_{\max }$ (solid line in Fig. A1). 
Appendix 1 (continued)

Fig. A1. The Michaelis-Menten (MM) approximation (broken line) versus the analytical expression (solid line) for the uptake rate (Eq. A1). The uptake rate was calculated from the estimated characteristics of Vibrio splendidus. A half-saturation constant, $K=V_{\max } / \alpha=\frac{\pi r(1-p)+n s}{4 h \pi D r s}$, was assumed for the MM approximation. See Fig. 1A for relative errors of this MM approximation

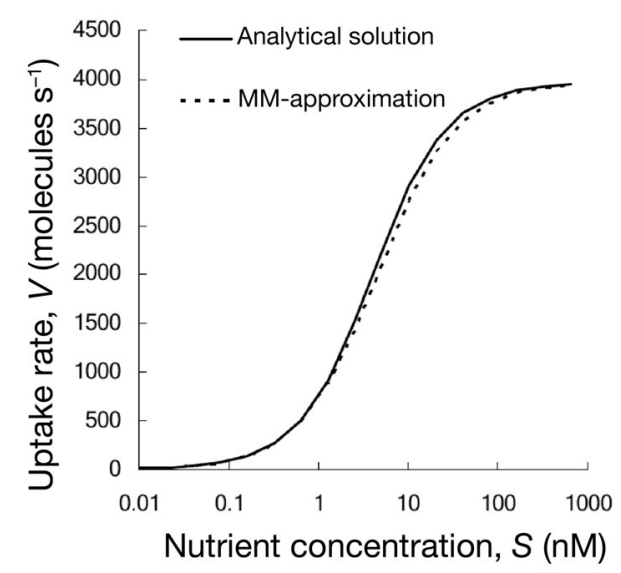

Editorial responsibility: Ronald Kiene, Mobile, Alabama, USA
Submitted: March 30, 2011; Accepted: August 20, 2011

Proofs received from author(s): October 23, 2011 